\title{
Taxonomy of the order Mononegavirales: update 2018
}

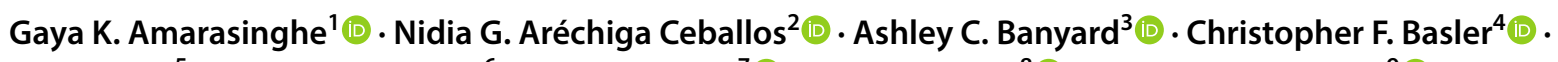
Sina Bavari ${ }^{5} \cdot$ Andrew J. Bennett $^{6} \cdot$ Kim R. Blasdell $^{7}$. Thomas Briese $^{8}$ (D) Alexander Bukreyev $^{9}$ (D) Yíngyún Caì $^{10}$ (1) Charles H. Calisher ${ }^{11}$ (D) Cristine Campos Lawson ${ }^{10} \cdot$ Kartik Chandran $^{12}$. Colin A. Chapman ${ }^{13,14,15}$.

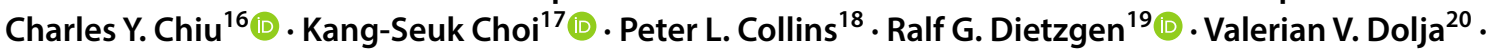

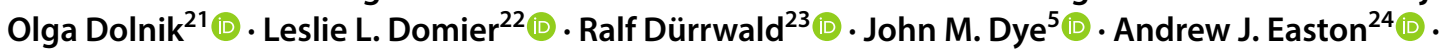

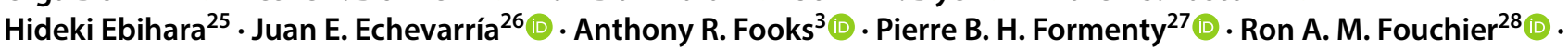

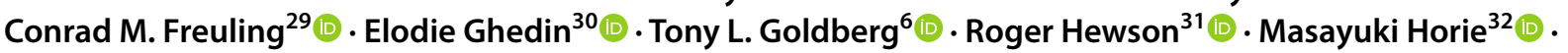
Timothy H. Hyndman ${ }^{33}$ (1) . Dàohóng Jiāng ${ }^{34}$ • Robert Kityo ${ }^{35}$. Gary P. Kobinger ${ }^{36}$. Hideki Kondō $^{37}$.

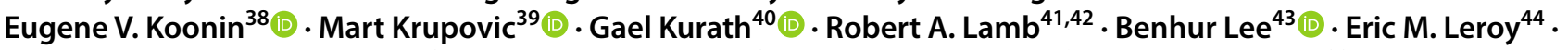
Piet Maes ${ }^{45}$ (1) Andrea Maisner ${ }^{21}$ (1) Denise A. Marston ${ }^{3}$ (1) . Sunil Kumar Mor ${ }^{46}$ (1) Thomas Müller $^{29}$ (D)

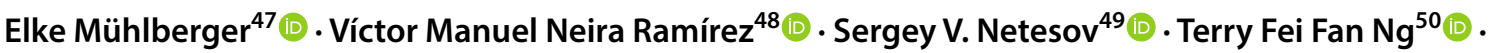

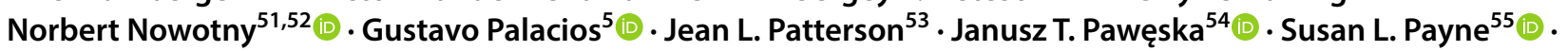
Karla Prieto ${ }^{5}$ - Bertus K. Rima ${ }^{56}$ - Paul Rota ${ }^{57}$. Dennis Rubbenstroth ${ }^{58} \cdot$ Martin Schwemmle $^{58} \cdot$ Stuart Siddell $^{59}$. Sophie J. Smither ${ }^{60}$. Qisheng Song ${ }^{61} \cdot$ Timothy Song $^{30} \cdot$ Mark D. Stenglein $^{62}$ (D) David M. Stone $^{63}$ (D) Ayato Takada $^{64}$ (1) Robert B. Tesh ${ }^{65} \cdot$ Luciano Matsumiya Thomazelli $^{66}\left(\right.$ Keizō Tomonaga $^{67} \cdot$ Noël Tordo $^{68,69}$.

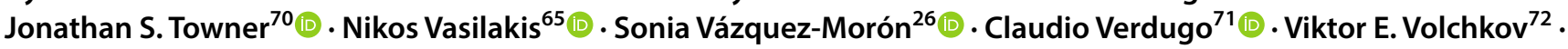
Victoria Wahl ${ }^{73}$ (1) Peter J. Walker ${ }^{74}$ (1) David Wang ${ }^{75} \cdot$ Lin-Fa Wang $^{76} \cdot$ James F. X. Wellehan $^{77}$ (1) Michael R. Wiley ${ }^{9,78}$ - Anna E. Whitfield ${ }^{79}$ - Yuri I. Wolf ${ }^{38} \cdot$ Gōngyín Yè $^{80} \cdot$ Yǒng-Zhèn Zhāng $^{81}$. Jens H. Kuhn ${ }^{10}$ (1)

Received: 5 March 2018 / Accepted: 12 March 2018 / Published online: 11 April 2018 (c) This is a U.S. government work and its text is not subject to copyright protection in the United States; however, its text may be subject to foreign copyright protection 2018

\begin{abstract}
In 2018, the order Mononegavirales was expanded by inclusion of 1 new genus and 12 novel species. This article presents the updated taxonomy of the order Mononegavirales as now accepted by the International Committee on Taxonomy of Viruses (ICTV) and summarizes additional taxonomic proposals that may affect the order in the near future.
\end{abstract}

Handling Editor: Sead Sabanadzovic.

Thomas Briese, Ralf Dürrwald, Masayuki Horie, Norbert Nowotny, Susan L. Payne, Dennis Rubbenstroth, Martin Schwemmle, Keizō Tomonaga and Jens H. Kuhn were the members of the 2014-2017 International Committee on Taxonomy of Viruses (ICTV) Bornaviridae Study Group; Gaya K. Amarasinghe, Christopher F. Basler, Sina Bavari, Alexander Bukreyev, Kartik Chandran, Olga Dolnik, John M. Dye, Hideki Ebihara, Pierre B. H. Formenty, Roger Hewson, Gary P. Kobinger, Eric M. Leroy, Elke Mühlberger, Sergey V. Netesov, Jean L. Patterson, Janusz T. Pawęska, Sophie J. Smither, Ayato Takada, Jonathan S. Towner, Viktor E. Volchkov, Victoria Wahl and Jens H. Kuhn were the members of the 2014-2017 ICTV Filoviridae Study Group; Ralf G. Dietzgen, Andrew J. Easton, Gael Kurath, Norbert Nowotny, Bertus K. Rima, Dennis Rubbenstroth, Nikos Vasilakis, Peter J. Walker and Jens H. Kuhn were the members of the 2014-2017 ICTV Mononegavirales Study Group; Ralf
G. Dietzgen, Leslie L. Domier, Elodie Ghedin, Dàohóng Jiāng, Nikos Vasilakis, David Wang and Jens H. Kuhn were the members of the 2014-2017 ICTV Nyamiviridae Study Group; Peter L. Collins, Andrew J. Easton, Ron A. M. Fouchier, Gael Kurath, Robert A. Lamb, Benhur Lee, Andrea Maisner, Bertus K. Rima, Paul Rota and Lin-Fa Wang were the members of the 2014-2017 ICTV Paramyxoviridae Study Group; Kim R. Blasdell, Charles H. Calisher, Ralf G. Dietzgen, Hideki Kondō, Gael Kurath, David M. Stone, Robert B. Tesh, Noël Tordo, Nikos Vasilakis, Peter J. Walker and Anna E. Whitfield are the members of the 2014-2017 ICTV Rhabdoviridae Study Group; and Stuart Siddell was the 2014-2017 ICTV Chair of the Animal dsRNA and ssRNAViruses Subcommittee.

Extended author information available on the last page of the article 


\section{Introduction}

The virus order Mononegavirales was established in 1991 to accommodate related viruses with nonsegmented, linear, single-stranded, negative-sense RNA genomes distributed among three families $[19,20]$. Today, the order includes 8 families [1, 2, 11, 21]. Amended/emended order descriptions were published in 1995 [5], 1997 [22], 2000 [23], 2005 [24], 2011 [6], 2016 [2], and 2017 [3]. In 2017, the Study Groups of the International Committee on Taxonomy of Viruses (ICTV) responsible for the taxonomy of the order and its 8 families assigned unclassified mononegaviruses to existing or novel taxa and continued efforts to streamline the order nomenclature in collaboration with other virus experts. Here we present the changes that were proposed via official ICTV taxonomic proposals (TaxoProps) at http://www.ictvonline.org/ in 2017 and accepted by the ICTV Executive Committee (EC). These changes are part of the official ICTV taxonomy as of 2018.

\section{Taxonomic changes at the order rank}

In 2018 , no changes were made at the order rank.

\section{Taxonomic changes at the family rank}

\section{Bornaviridae}

The family Bornaviridae was expanded in 2018 by creation of a second genus (Carbovirus), including two novel species for the newly discovered jungle carpet python virus (JCPV) and southwest carpet python virus (SWCPV) found in carpet pythons (Pythonidae: Morelia spilota), respectively [10]. The previously established genus Bornavirus was renamed Orthobornavirus to remove the ambiguity of the terms "bornavirus"/"bornaviral" that resulted due to the creation of the second genus (in absence of the genus name change, "bornavirus"/"bornaviral" could refer either to all members of the family Bornaviridae or only to those of the genus Bornavirus). All binomial species names of the genus Bornavirus were adjusted by replacing the genus epithet "bornavirus" with "orthobornavirus" (TaxoProps 2017.005M.A.v1. Carbovirus and 2017.004M.A.v1.Bornaviridae_ren).

\section{Filoviridae}

The species name Taï Forest ebolavirus was changed to Tai Forest ebolavirus by removal of the diaeresis (TaxoProp 2017.001G.A.v2.43spren).

\section{Mymonaviridae}

In 2018 , no changes were made at the family rank.

\section{Nyamiviridae}

In 2018, no changes were made at the family rank.

\section{Paramyxoviridae}

The genus Avulavirus was expanded in 2018 by the addition of six species (TaxoProp 2017.010M.A.v2.Avulavirus_6sp). The species Avian avulavirus 14-16 were established for avian paramyxoviruses 14-16 (APMV-14-16) that were recently discovered in an unspecified duck in Japan, a whiterumped sandpiper (Scolopacidae: Calidris fuscicollis) in Brazil, and unspecified birds in South Korea, respectively [12, 27, 28]. The species Avian avulavirus 17-19 were established for Antarctic penguin viruses A-C (APVA-APVC) that were recently discovered in Antarctic long-tailed gentoo penguins (Spheniscidae: Pygoscelis papua) [18].

\section{Pneumoviridae}

In 2018, no changes were made at the family rank.

\section{Rhabdoviridae}

The genus Ledantevirus was expanded in 2018 by one species, Kanywara ledantevirus, for Kanywara virus (KYAV) recently discovered in an unclassified nycteribiid batfly in Uganda [8] (TaxoProp 2017.009M.A.v1.Ledantevirus_sp).

The genus Lyssavirus was expanded by the addition of two novel species, Gannoruwa bat lyssavirus and Lleida bat lyssavirus, for Gannoruwa bat lyssavirus (GBLV) and Lleida bat virus (LLEBV), recently discovered in Indian flying foxes (Pteropus medius) in Sri Lanka and in a Schreibers's long-fingered bat (Miniopterus schreibersii) in Spain in 2011, respectively [4, 9, 15] (TaxoProps 2017.013M.A.v1.Lyssavirus_sp and 2017.014M.A.v1. Lyssavirus_sp).

Finally, the genus Tibrovirus was expanded by one species, Beatrice Hill tibrovirus, for Beatrice Hill virus (BHV) discovered in 1984 in Australian biting midges (Ceratopogonidae: Culicoides peregrinus) [26, 29] (TaxoProp 2017.019M.U.v1.Tibrovirus_sp).

\section{Sunviridae}

In 2018 , no changes were made at the family rank. 
Table 1 ICTV-accepted taxonomy of the order Mononegavirales as of 2018. Listed are all mononegaviruses that have been classified into species

\begin{tabular}{|c|c|c|}
\hline Genus & Species $^{\mathbb{I}}$ & Virus (Abbreviation) $)^{\mathbb{I}}$ \\
\hline \multicolumn{3}{|l|}{ Family Bornaviridae } \\
\hline \multirow[t]{2}{*}{ Carbovirus } & Queensland carbovirus* & jungle carpet python virus (JCPV) \\
\hline & Southwest carbovirus & southwest carpet python virus (SWCPV) \\
\hline \multirow[t]{16}{*}{ Orthobornavirus } & Elapid 1 orthobornavirus & Loveridge's garter snake virus 1 (LGSV-1) \\
\hline & Mammalian 1 orthobornavirus* & Borna disease virus 1 (BoDV-1) \\
\hline & & Borna disease virus 2 (BoDV-2) \\
\hline & Mammalian 2 orthobornavirus & variegated squirrel bornavirus 1 (VSBV-1) \\
\hline & Passeriform 1 orthobornavirus & canary bornavirus $1(\mathrm{CnBV}-1)$ \\
\hline & & canary bornavirus $2(\mathrm{CnBV}-2)$ \\
\hline & & canary bornavirus 3 (CnBV-3) \\
\hline & Passeriform 2 orthobornavirus & estrildid finch bornavirus 1 (EsBV-1) \\
\hline & Psittaciform 1 orthobornavirus & parrot bornavirus 1 (PaBV-1) \\
\hline & & parrot bornavirus $2(\mathrm{PaBV}-2)$ \\
\hline & & parrot bornavirus 3 (PaBV-3) \\
\hline & & parrot bornavirus 4 (PaBV-4) \\
\hline & & parrot bornavirus 7 (PaBV-7) \\
\hline & Psittaciform 2 orthobornavirus & parrot bornavirus 5 (PaBV-5) \\
\hline & Waterbird 1 orthobornavirus & aquatic bird bornavirus 1 (ABBV-1) \\
\hline & & aquatic bird bornavirus 2 (ABBV-2) \\
\hline \multicolumn{3}{|l|}{ Family Filoviridae } \\
\hline Cuevavirus & Lloviu cuevavirus* & Lloviu virus (LLOV) \\
\hline \multirow[t]{5}{*}{ Ebolavirus } & Bundibugyo ebolavirus & Bundibugyo virus (BDBV) \\
\hline & Reston ebolavirus & Reston virus (RESTV) \\
\hline & Sudan ebolavirus & Sudan virus (SUDV) \\
\hline & Tai Forest ebolavirus & Taï Forest virus (TAFV) \\
\hline & Zaire ebolavirus* & Ebola virus (EBOV) \\
\hline \multirow[t]{2}{*}{ Marburgvirus } & Marburg marburgvirus* & Marburg virus (MARV) \\
\hline & & Ravn virus (RAVV) \\
\hline \multicolumn{3}{|l|}{ Family Mymonaviridae } \\
\hline Sclerotimonavirus & Sclerotinia sclerotimonavirus* & $\begin{array}{l}\text { Sclerotinia sclerotiorum negative-stranded RNA virus } 1 \\
\text { (SsNSRV-1) }\end{array}$ \\
\hline \multicolumn{3}{|l|}{ Family Nyamiviridae } \\
\hline \multirow[t]{3}{*}{ Nyavirus } & Midway nyavirus & Midway virus (MIDWV) \\
\hline & Nyamanini nyavirus* & Nyamanini virus (NYMV) \\
\hline & Sierra Nevada nyavirus & Sierra Nevada virus (SNVV) \\
\hline Peropuvirus & Pteromalus puparum peropuvirus* & $\begin{array}{l}\text { Pteromalus puparum negative-strand RNA virus } 1 \\
\text { (PpNSRV-1) }\end{array}$ \\
\hline Socyvirus & Soybean cyst nematode socyvirus* & soybean cyst nematode virus 1 (SbCNV-1) \\
\hline \multicolumn{3}{|c|}{ Family Paramyxoviridae } \\
\hline Aquaparamyxovirus & Salmon aquaparamyxovirus* & Atlantic salmon paramyxovirus (AsaPV) \\
\hline \multirow[t]{7}{*}{ Avulavirus } & Avian avulavirus $1 *$ & avian paramyxovirus $1(\mathrm{APMV}-1)^{1}$ \\
\hline & Avian avulavirus 2 & avian paramyxovirus 2 (APMV-2) \\
\hline & Avian avulavirus 3 & avian paramyxovirus 3 (APMV-3) \\
\hline & Avian avulavirus 4 & avian paramyxovirus 4 (APMV-4) \\
\hline & Avian avulavirus 5 & avian paramyxovirus 5 (APMV-5) \\
\hline & Avian avulavirus 6 & avian paramyxovirus 6 (APMV-6) \\
\hline & Avian avulavirus 7 & avian paramyxovirus 7 (APMV-7) \\
\hline
\end{tabular}


Table 1 (continued)

\begin{tabular}{|c|c|c|}
\hline Genus & Species $^{\text {II }}$ & Virus (Abbreviation) $)^{\mathbb{I}}$ \\
\hline & Avian avulavirus 8 & avian paramyxovirus 8 (APMV-8) \\
\hline & Avian avulavirus 9 & avian paramyxovirus 9 (APMV-9) \\
\hline & Avian avulavirus 10 & avian paramyxovirus 10 (APMV-10) \\
\hline & Avian avulavirus 11 & avian paramyxovirus 11 (APMV-11) \\
\hline & Avian avulavirus 12 & avian paramyxovirus 12 (APMV-12) \\
\hline & Avian avulavirus 13 & avian paramyxovirus 13 (APMV-13) \\
\hline & Avian avulavirus 14 & avian paramyxovirus 14 (APMV-14) \\
\hline & Avian avulavirus 15 & avian paramyxovirus 15 (APMV-15) \\
\hline & Avian avulavirus 16 & avian paramyxovirus 16 (APMV-16) \\
\hline & Avian avulavirus 17 & Antarctic penguin virus A (APV-A) \\
\hline & Avian avulavirus 18 & Antarctic penguin virus B (APV-B) \\
\hline & Avian avulavirus 19 & Antarctic penguin virus C (APV-C) \\
\hline Ferlavirus & Reptilian ferlavirus* & Fer-de-Lance virus (FDLV) \\
\hline \multirow[t]{5}{*}{ Henipavirus } & Cedar henipavirus & Cedar virus $(\mathrm{CedV})$ \\
\hline & Ghanaian bat henipavirus & Kumasi virus $(\mathrm{KV})^{2}$ \\
\hline & Hendra henipavirus* & Hendra virus $(\mathrm{HeV})$ \\
\hline & Mojiang henipavirus & Mòjiāng virus (MojV) \\
\hline & Nipah henipavirus & Nipah virus (NiV) \\
\hline \multirow[t]{7}{*}{ Morbillivirus } & Canine morbillivirus & canine distemper virus (CDV) \\
\hline & Cetacean morbillivirus & cetacean morbillivirus (CeMV) \\
\hline & Feline morbillivirus & feline morbillivirus (FeMV) \\
\hline & Measles morbillivirus* & measles virus $(\mathrm{MeV})$ \\
\hline & Small ruminant morbillivirus & peste-des-petits-ruminants virus (PPRV) \\
\hline & Phocine morbillivirus & phocine distemper virus (PDV) \\
\hline & Rinderpest morbillivirus & rinderpest virus (RPV) \\
\hline \multirow[t]{5}{*}{ Respirovirus } & Bovine respirovirus 3 & bovine parainfluenza virus 3 (BPIV-3) \\
\hline & Human respirovirus 1 & human parainfluenza virus 1 (HPIV-1) \\
\hline & Human respirovirus 3 & human parainfluenza virus 3 (HPIV-3) \\
\hline & Porcine respirovirus 1 & porcine parainfluenza virus 1 (PPIV-1) \\
\hline & Murine respirovirus* & Sendai virus $(\mathrm{SeV})^{3}$ \\
\hline \multirow{18}{*}{ Rubulavirus } & Achimota rubulavirus 1 & Achimota virus 1 (AchPV-1) \\
\hline & Achimota rubulavirus 2 & Achimota virus 2 (AchPV-2) \\
\hline & Bat mumps rubulavirus & bat mumps virus $(\mathrm{BMV})^{4}$ \\
\hline & Canine rubulavirus & parainfluenza virus $5(\mathrm{PIV}-5)^{5}$ \\
\hline & Human rubulavirus 2 & human parainfluenza virus 2 (HPIV-2) \\
\hline & Human rubulavirus 4 & human parainfluenza virus 4a (HPIV-4a) \\
\hline & & human parainfluenza virus 4b (HPIV-4b) \\
\hline & Mapuera rubulavirus & Mapuera virus (MapV) \\
\hline & Menangle rubulavirus & Menangle virus (MenPV) \\
\hline & Mumps rubulavirus* & mumps virus $(\mathrm{MuV})$ \\
\hline & Porcine rubulavirus & La Piedad Michoacán Mexico virus (LPMV) ${ }^{6}$ \\
\hline & Simian rubulavirus & simian virus $41(\mathrm{SV}-41)$ \\
\hline & Sosuga rubulavirus & Sosuga virus \\
\hline & Teviot rubulavirus & Teviot virus (TevPV) \\
\hline & Tioman rubulavirus & Tioman virus (TioPV) \\
\hline & Tuhoko rubulavirus 1 & Tuhoko virus 1 (ThkPV-1) \\
\hline & Tuhoko rubulavirus 2 & Tuhoko virus 2 (ThkPV-2) \\
\hline & Tuhoko rubulavirus 3 & Tuhoko virus 3 (ThkPV-3) \\
\hline
\end{tabular}


Table 1 (continued)

\begin{tabular}{|c|c|c|}
\hline Genus & Species $^{\text {II }}$ & Virus (Abbreviation) $^{\mathrm{I}}$ \\
\hline \multicolumn{3}{|c|}{ Family Pneumoviridae } \\
\hline \multirow[t]{2}{*}{ Metapneumovirus } & Avian metapneumovirus $*$ & avian metapneumovirus (AMPV) ${ }^{7}$ \\
\hline & Human metapneumovirus & human metapneumovirus (HMPV) \\
\hline \multirow[t]{4}{*}{ Orthopneumovirus } & Bovine orthopneumovirus & bovine respiratory syncytial virus (BRSV) \\
\hline & Human orthopneumovirus* & human respiratory syncytial virus A2 (HRSV-A2) \\
\hline & & human respiratory syncytial virus B1 (HRSV-B1) \\
\hline & Murine orthopneumovirus & murine pneumonia virus (MPV) \\
\hline \multicolumn{3}{|l|}{ Family Rhabdoviridae } \\
\hline \multirow[t]{5}{*}{ Almendravirus } & Arboretum almendravirus & Arboretum virus (ABTV) \\
\hline & Balsa almendravirus & Balsa virus (BALV) \\
\hline & Coot Bay almendravirus & Coot Bay virus (CBV) \\
\hline & Puerto Almendras almendravirus* & Puerto Almendras virus (PTAMV) \\
\hline & Rio Chico almendravirus & Rio Chico virus (RCHV) \\
\hline \multirow[t]{4}{*}{ Curiovirus } & Curionopolis curiovirus $*$ & Curionopolis virus (CURV) \\
\hline & Iriri curiovirus & Iriri virus (IRIRV) \\
\hline & Itacaiunas curiovirus & Itacaiunas virus (ITAV) \\
\hline & Rochambeau curiovirus & Rochambeau virus (RBUV) \\
\hline \multirow[t]{11}{*}{ Cytorhabdovirus } & Alfalfa dwarf cytorhabdovirus & alfalfa dwarf virus (ADV) \\
\hline & Barley yellow striate mosaic cytorhabdovirus & barley yellow striate mosaic virus (BYSMV) \\
\hline & Broccoli necrotic yellows cytorhabdovirus & broccoli necrotic yellows virus (BNYV) \\
\hline & Colocasia bobone disease-associated cytorhabdovirus & Colocasia bobone disease-associated virus ( $\mathrm{CBDaV})$ \\
\hline & Festuca leaf streak cytorhabdovirus & festuca leaf streak virus (FLSV) \\
\hline & Lettuce necrotic yellows cytorhabdovirus* & lettuce necrotic yellows virus (LNYV) \\
\hline & Lettuce yellow mottle cytorhabdovirus & lettuce yellow mottle virus (LYMoV) \\
\hline & Northern cereal mosaic cytorhabdovirus & northern cereal mosaic virus (NCMV) \\
\hline & Sonchus cytorhabdovirus 1 & sonchus virus (SonV) \\
\hline & Strawberry crinkle cytorhabdovirus & strawberry crinkle virus (SCV) \\
\hline & Wheat American striate mosaic cytorhabdovirus & wheat American striate mosaic virus (WASMV) \\
\hline \multirow[t]{2}{*}{ Dichorhavirus } & Coffee ringspot dichorhavirus & coffee ringspot virus (CoRSV) \\
\hline & Orchid fleck dichorhavirus* & orchid fleck virus $(\mathrm{OFV})^{8}$ \\
\hline \multirow[t]{9}{*}{ Ephemerovirus } & Adelaide River ephemerovirus & Adelaide River virus (ARV) \\
\hline & Berrimah ephemerovirus & Berrimah virus (BRMV) \\
\hline & Bovine fever ephemerovirus* & bovine ephemeral fever virus (BEFV) \\
\hline & Kimberley ephemerovirus & Kimberley virus (KIMV) \\
\hline & & Malakal virus (MALV) \\
\hline & Koolpinyah ephemerovirus & Koolpinyah virus (KOOLV) \\
\hline & Kotonkan ephemerovirus & kotonkan virus (KOTV) \\
\hline & Obodhiang ephemerovirus & Obodhiang virus (OBOV) \\
\hline & Yata ephemerovirus & Yata virus (YATV) \\
\hline \multirow[t]{10}{*}{ Hapavirus } & Flanders hapavirus & Flanders virus (FLAV) \\
\hline & Hart Park hapavirus & Hart Park virus (HPV) \\
\hline & Gray Lodge hapavirus & Gray Lodge virus (GLOV) \\
\hline & Joinjakaka hapavirus & Joinjakaka virus (JOIV) \\
\hline & La Joya hapavirus & La Joya virus (LJV) \\
\hline & Kamese hapavirus & Kamese virus (KAMV) \\
\hline & Landjia hapavirus & Landjia virus (LANV = LJAV) \\
\hline & Manitoba hapavirus & Manitoba virus (MANV = MNTBV) \\
\hline & Marco hapavirus & Marco virus (MCOV) \\
\hline & Mosqueiro hapavirus & Mosqueiro virus (MQOV) \\
\hline
\end{tabular}


Table 1 (continued)

\begin{tabular}{|c|c|c|}
\hline Genus & Species $^{\mathbb{I}}$ & Virus (Abbreviation) $)^{\mathbb{I}}$ \\
\hline & Mossuril hapavirus & Mossuril virus (MOSV) \\
\hline & Ngaingan hapavirus & Ngaingan virus (NGAV) \\
\hline & Ord River hapavirus & Ord River virus (ORV) \\
\hline & Parry Creek hapavirus & Parry Creek virus (PCV) \\
\hline & Wongabel hapavirus* & Wongabel virus (WONV) \\
\hline \multirow[t]{15}{*}{ Ledantevirus } & Barur ledantevirus & Barur virus (BARV) \\
\hline & Fikirini ledantevirus & Fikirini virus (FKRV) \\
\hline & Fukuoka ledantevirus & Fukuoka virus (FUKV) \\
\hline & Kanyawara ledantevirus & Kanyawara virus (KYAV) \\
\hline & Kern Canyon ledantevirus & Kern Canyon virus (KCV) \\
\hline & Keuraliba ledantevirus & Keuraliba virus (KEUV) \\
\hline & Kolente ledantevirus & Kolente virus (KOLEV) \\
\hline & Kumasi ledantevirus & Kumasi rhabdovirus (KRV) \\
\hline & Le Dantec ledantevirus* & Le Dantec virus (LDV) \\
\hline & Mount Elgon bat ledantevirus & Mount Elgon bat virus (MEBV) \\
\hline & Nkolbisson ledantevirus & Nkolbisson virus (NKOV) \\
\hline & Nishimuro ledantevirus & Nishimuro virus (NISV) ${ }^{9}$ \\
\hline & Oita ledantevirus & Oita virus (OITAV) \\
\hline & Wuhan ledantevirus & Wǔhàn louse fly virus 5 (WLFV-5) \\
\hline & Yongjia ledantevirus & Yǒngjiā tick virus 2 (YTV-2) \\
\hline \multirow[t]{16}{*}{ Lyssavirus } & Aravan lyssavirus & Aravan virus (ARAV) \\
\hline & Australian bat lyssavirus & Australian bat lyssavirus (ABLV) \\
\hline & Bokeloh bat lyssavirus & Bokeloh bat lyssavirus (BBLV) \\
\hline & Duvenhage lyssavirus & Duvenhage virus (DUVV) \\
\hline & European bat 1 lyssavirus & European bat lyssavirus 1 (EBLV-1) \\
\hline & European bat 2 lyssavirus & European bat lyssavirus 2 (EBLV-2) \\
\hline & Gannoruwa bat lyssavirus & Gannoruwa bat lyssavirus (GBLV) \\
\hline & Ikoma lyssavirus & Ikoma lyssavirus (IKOV) \\
\hline & Irkut lyssavirus & Irkut virus (IRKV) \\
\hline & Khujand lyssavirus & Khujand virus (KHUV) \\
\hline & Lagos bat lyssavirus & Lagos bat virus (LBV) \\
\hline & Lleida bat lyssavirus & Lleida bat virus (LLEBV) \\
\hline & Mokola lyssavirus & Mokola virus (MOKV) \\
\hline & Rabies lyssavirus* & rabies virus $(\mathrm{RABV})$ \\
\hline & Shimoni bat lyssavirus & Shimoni bat virus (SHIBV) \\
\hline & West Caucasian bat lyssavirus & West Caucasian bat virus (WCBV) \\
\hline \multirow[t]{4}{*}{ Novirhabdovirus } & Hirame novirhabdovirus & hirame rhabdovirus (HIRV) \\
\hline & Piscine novirhabdovirus & viral hemorrhagic septicemia virus $(\mathrm{VHSV})^{10}$ \\
\hline & Salmonid novirhabdovirus* & infectious hematopoietic necrosis virus (IHNV) \\
\hline & Snakehead novirhabdovirus & snakehead rhabdovirus (SHRV) \\
\hline \multirow[t]{9}{*}{ Nucleorhabdovirus } & Datura yellow vein nucleorhabdovirus & datura yellow vein virus (DYVV) \\
\hline & Eggplant mottled dwarf nucleorhabdovirus & eggplant mottled dwarf virus (EMDV) \\
\hline & Maize fine streak nucleorhabdovirus & maize fine streak virus (MSFV) \\
\hline & Maize Iranian mosaic nucleorhabdovirus & maize Iranian mosaic virus (MIMV) \\
\hline & Maize mosaic nucleorhabdovirus & maize mosaic virus (MMV) \\
\hline & Potato yellow dwarf nucleorhabdovirus* & potato yellow dwarf virus (PYDV) \\
\hline & Rice yellow stunt nucleorhabdovirus & rice yellow stunt virus (RYSV) \\
\hline & & rice transitory yellowing virus (RTYV) \\
\hline & Sonchus yellow net nucleorhabdovirus & sonchus yellow net virus (SYNV) \\
\hline
\end{tabular}


Table 1 (continued)

\begin{tabular}{|c|c|c|}
\hline Genus & Species ${ }^{\mathbb{I}}$ & Virus (Abbreviation) $)^{\mathbb{I}}$ \\
\hline & Sowthistle yellow vein nucleorhabdovirus & sowthistle yellow vein virus (SYVV) \\
\hline & Taro vein chlorosis nucleorhabdovirus & taro vein chlorosis virus (TaVCV) \\
\hline \multirow[t]{3}{*}{ Perhabdovirus } & Anguillid perhabdovirus & eel virus European X (EVEX) \\
\hline & Perch perhabdovirus* & perch rhabdovirus (PRV) \\
\hline & Sea trout perhabdovirus & lake trout rhabdovirus (LTRV) \\
\hline \multirow[t]{7}{*}{ Sigmavirus } & Drosophila affinis sigmavirus & Drosophila affinis sigmavirus (DAffSV) \\
\hline & Drosophila ananassae sigmavirus & Drosophila ananassae sigmavirus (DAnaSV) \\
\hline & Drosophila immigrans sigmavirus & Drosophila immigrans sigmavirus (DImmSV) \\
\hline & Drosophila melanogaster sigmavirus $*$ & Drosophila melanogaster sigmavirus (DMelSV) \\
\hline & Drosophila obscura sigmavirus & Drosophila obscura sigmavirus (DObsSV) \\
\hline & Drosophila tristis sigmavirus & Drosophila tristis sigmavirus (DTriSV) \\
\hline & Muscina stabulans sigmavirus & Muscina stabulans sigmavirus (MStaSV) \\
\hline \multirow[t]{4}{*}{ Sprivivirus } & Carp sprivivirus* & spring viremia of carp virus (SVCV) \\
\hline & Pike fry sprivivirus & grass carp rhabdovirus (GrCRV) \\
\hline & & pike fry rhabdovirus (PFRV) \\
\hline & & tench rhabdovirus (TenRV) \\
\hline \multirow[t]{5}{*}{ Sripuvirus } & Almpiwar sripuvirus & Almpiwar virus (ALMV) \\
\hline & Chaco sripuvirus & Chaco virus $(\mathrm{CHOV})$ \\
\hline & Niakha sripuvirus* & Niakha virus (NIAV) \\
\hline & Sena Madureira sripuvirus & Sena Madureira virus (SMV) \\
\hline & Sripur sripuvirus & Sripur virus (SRIV) \\
\hline \multirow[t]{8}{*}{ Tibrovirus } & Bas Congo tibrovirus & Bas-Congo virus (BASV) \\
\hline & Beatrice Hill tibrovirus & Beatrice Hill virus (BHV) \\
\hline & Coastal Plains tibrovirus & Coastal Plains virus (CPV) \\
\hline & Ekpoma 1 tibrovirus & Ekpoma virus 1 (EKV-1) \\
\hline & Ekpoma 2 tibrovirus & Ekpoma virus 2 (EKV-2) \\
\hline & Sweetwater Branch tibrovirus & Sweetwater Branch virus (SWBV) \\
\hline & Tibrogargan tibrovirus* & Bivens Arm virus (BAV) \\
\hline & & Tibrogargan virus (TIBV) \\
\hline \multirow[t]{3}{*}{ Tupavirus } & Durham tupavirus* & Durham virus (DURV) \\
\hline & Klamath tupavirus & Klamath virus (KLAV) \\
\hline & Tupaia tupavirus & tupaia virus (TUPV) \\
\hline Varicosavirus & Lettuce big-vein associated varicosavirus* & lettuce big-vein associated virus $(\mathrm{LBVaV})^{11}$ \\
\hline \multirow[t]{16}{*}{ Vesiculovirus } & Alagoas vesiculovirus & vesicular stomatitis Alagoas virus (VSAV) \\
\hline & American bat vesiculovirus & American bat vesiculovirus (ABVV) \\
\hline & Carajas vesiculovirus & Carajás virus (CJSV) \\
\hline & Chandipura vesiculovirus & Chandipura virus (CHPV) \\
\hline & Cocal vesiculovirus & Cocal virus $(\mathrm{COCV})$ \\
\hline & Indiana vesiculovirus* & vesicular stomatitis Indiana virus (VSIV) \\
\hline & Isfahan vesiculovirus & Isfahan virus (ISFV) \\
\hline & Jurona vesiculovirus & Jurona virus (JURV) \\
\hline & Malpais Spring vesiculovirus & Malpais Spring virus (MSPV) \\
\hline & Maraba vesiculovirus & Maraba virus (MARAV) \\
\hline & Morreton vesiculovirus & Morreton virus (MORV) \\
\hline & New Jersey vesiculovirus & vesicular stomatitis New Jersey virus (VSNJV) \\
\hline & Perinet vesiculovirus & Perinet virus (PERV) \\
\hline & Piry vesiculovirus & Piry virus (PIRYV) \\
\hline & Radi vesiculovirus & Radi virus (RADV) \\
\hline & Yug Bogdanovac vesiculovirus & Yug Bogdanovac virus (YBV) \\
\hline
\end{tabular}


Table 1 (continued)

\begin{tabular}{lll}
\hline Genus & Species $^{\mathbb{I}}$ & Virus (Abbreviation) $^{\mathbb{I}}$ \\
\hline Unassigned & Moussa virus & Moussa virus (MOUV) \\
$\begin{array}{l}\text { Family Sunviridae } \\
\text { Sunshinevirus }\end{array}$ & Reptile sunshinevirus $1^{*}$ & Sunshine Coast virus (SunCV) \\
Unassigned & & \\
Anphevirus & Xincheng anphevirus* & Xīnchéng mosquito virus (XcMV) \\
Arlivirus & Lishi arlivirus* & Líshí spider virus 2 (LsSV-2) \\
Chengtivirus & Tacheng chengtivirus* & Tăchéng tick virus 6 (TcTV-6) \\
Crustavirus & Wenzhou crustavirus* & Wēnzhōu crab virus 1 (WzCV-1) \\
Wastrivirus & Sanxia wastrivirus* & Sānxiá water strider virus 4 (SxWSV-4) \\
\hline
\end{tabular}

*Asterisks denote type species. "IPlease note that viruses are real objects that are assigned to concepts that are called taxa. Species, genera, families, and orders are taxa. Taxon names are always italicized and always begin with a capital letter. Virus names, on the other hand, are not italicized and are not capitalized, except if the name or a name component is a proper noun. This column lists the virus names with their correct (lack of) capitalization

${ }^{1}$ Includes: Newcastle disease virus (NDV) and pigeon paramyxovirus 1 (PPMV-1); ${ }^{2}$ synonym: GH-M74a virus; ${ }^{3}$ synonym: murine parainfluenza virus 1 ; ${ }^{4}$ synonym: bat paramyxovirus; ${ }^{5}$ synonym: simian virus $5 ;{ }^{6}$ synonym: porcine rubulavirus; ${ }^{7}$ synonyms: avian pneumovirus, turkey rhinotracheitis virus; ${ }^{8}$ synonyms: citrus leprosis virus nuclear type, citrus necrotic spot virus; ${ }^{9}$ synonym: wild boar rhabdovirus 1 (WBRV1);

${ }^{10}$ synonyms: Egtved virus, Paralichthys olivaceus rhabdovirus; ${ }^{11}$ synonym: tobacco stunt virus

\section{Outlook}

The taxonomy of viruses of the order Mononegavirales remains in flux and additional important changes are likely forthcoming. Indeed, in 2017, two additional taxonomic proposals that would affect the order Mononegavirales were debated during the most recent ICTV EC meeting in Singapore. TaxoProp 2017.006M.U.v2.Negarnaviricota proposes

- establishment of a phylum for negative-sense RNA viruses that is subdivided into two subphyla;

- establishment of a sister order to the order Mononegavirales to accommodate the recently discovered "chǔviruses" $[13,25]$; and

- combination of both sister orders in a class assigned to one of the subphyla.

TaxoProp 2017.016M.U.v3.Mononegavirales_rev proposes

- expansion of the family Mymonaviridae by 6 species for novel soybean leaf-associated viruses and an invertebrate virus [16, 17, 25];

- transfer of the nyamiviral genus Peropuvirus into a new family and expansion of the genus by 6 species for novel invertebrate viruses $[13,25]$;

- transfer of the free-floating genus Crustavirus into the family Nyamiviridae; the expansion of Crustavirus by two species for novel invertebrate viruses; and the expansion of Nyamiviridae by an additional three genera that include a total of five species for novel invertebrate viruses $[13,25]$;

- establishment of a new family that absorbs the previously free-floating genus Anphevirus and expansion of this genus by six species for novel invertebrate viruses $[7,13,14,25]$; and

- dissolution of the previously free-floating genera Chengivirus and Wastrivirus and transfer of their species into the genus Arlivirus, expansion of Arlivirus by three new species for novel invertebrate viruses [13, 25], and transfer of this genus into a new family.

These two proposals failed to find unanimous approval at a final ICTV EC vote in fall of 2017 and were deferred to the 2018 ICTV EC meeting, at which a simple majority vote would suffice for approval of the original proposals.

\section{Summary}

A summary of the current, ICTV-accepted taxonomy of the order Mononegavirales is presented in Table 1.

Acknowledgements We thank Laura Bollinger (NIH/NIAID Integrated Research Facility at Fort Detrick, Frederick, MD, USA) for critically editing the manuscript.

Funding This work was supported in part through Battelle Memorial Institute's prime contract with the US National Institute of Allergy and Infectious Diseases (NIAID) under Contract no. HHSN272200700016I (Y.C., J.H.K.). This work was also funded in part under Contract no. HSHQDC-15-C-00064 awarded by DHS S\&T for the management and operation of the National Biodefense Analysis and 
Countermeasures Center (NBACC), a federally funded research and development center (V.W.); National Institutes of Health (NIH) contract HHSN272201000040I/HHSN27200004/D04 and Grant R24AI120942 (N.V., R.B.T.); and intramural funds of the US Department of Health and Human Services to the US National Library of Medicine (E.V.K. and Y.I.W.). This work was also supported by the UK Department for Environment, Food and Rural Affairs (Defra), Scottish Government and Welsh Government (Grant no. SV3500) (A.R.F.).

\section{Compliance with ethical standards}

The views and conclusions contained in this document are those of the authors and should not be interpreted as necessarily representing the official policies, either expressed or implied, of the US Department of the Army, the US Department of Defense, the US Department of Health and Human Services, the Department of Homeland Security (DHS) Science and Technology Directorate (S\&T), or of the institutions and companies affiliated with the authors. In no event shall any of these entities have any responsibility or liability for any use, misuse, inability to use, or reliance upon the information contained herein. The US departments do not endorse any products or commercial services mentioned in this publication.

Conflict of interest The authors declare no conflicts of interest.

Ethical approval This article does not contain any studies with human participants or animals performed by any of the authors.

\section{References}

1. Adams MJ, Lefkowitz EJ, King AM, Carstens EB (2014) Ratification vote on taxonomic proposals to the International Committee on Taxonomy of Viruses (2014). Arch Virol 159:2831-2841

2. Afonso $\mathrm{CL}$, Amarasinghe GK, Bányai $\mathrm{K}$, Bào $\mathrm{Y}$, Basler $\mathrm{CF}$, Bavari S, Bejerman N, Blasdell KR, Briand F-X, Briese T, Bukreyev A, Calisher CH, Chandran K, Chéng J, Clawson AN, Collins PL, Dietzgen RG, Dolnik O, Domier LL, Dürrwald R, Dye JM, Easton AJ, Ebihara H, Farkas SL, Freitas-Astúa J, Formenty P, Fouchier RA, Fù Y, Ghedin E, Goodin MM, Hewson R, Horie M, Hyndman TH, Jiāng D, Kitajima EW, Kobinger GP, Kondo H, Kurath G, Lamb RA, Lenardon S, Leroy EM, Li C-X, Lin X-D, Liú L, Longdon B, Marton S, Maisner A, Mühlberger E, Netesov SV, Nowotny N, Patterson JL, Payne SL, Paweska JT, Randall RE, Rima BK, Rota P, Rubbenstroth D, Schwemmle M, Shi M, Smither SJ, Stenglein MD, Stone DM, Takada A, Terregino C, Tesh RB, Tian J-H, Tomonaga K, Tordo N, Towner JS, Vasilakis N, Verbeek M, Volchkov VE, Wahl-Jensen V, Walsh JA, Walker PJ, Wang D, Wang L-F, Wetzel T, Whitfield AE, Xiè JT, Yuen K-Y, Zhang Y-Z, Kuhn JH (2016) Taxonomy of the order Mononegavirales: update 2016. Arch Virol 161:2351-2360

3. Amarasinghe GK, Bào Y, Basler CF, Bavari S, Beer M, Bejerman N, Blasdell KR, Bochnowski A, Briese T, Bukreyev A, Calisher $\mathrm{CH}$, Chandran K, Collins PL, Dietzgen RG, Dolnik O, Dürrwald R, Dye JM, Easton AJ, Ebihara H, Fang Q, Formenty P, Fouchier RAM, Ghedin E, Harding RM, Hewson R, Higgins CM, Hong J, Horie M, James AP, Jiāng D, Kobinger GP, Kondo H, Kurath G, Lamb RA, Lee B, Leroy EM, Li M, Maisner A, Mühlberger E, Netesov SV, Nowotny N, Patterson JL, Payne SL, Paweska JT, Pearson MN, Randall RE, Revill PA, Rima BK, Rota P, Rubbenstroth D, Schwemmle M, Smither SJ, Song Q, Stone DM, Takada A, Terregino C, Tesh RB, Tomonaga K, Tordo N, Towner JS,
Vasilakis N, Volchkov VE, Wahl-Jensen V, Walker PJ, Wang B, Wang D, Wang F, Wang L-F, Werren JH, Whitfield AE, Yan Z, Ye G, Kuhn JH (2017) Taxonomy of the order Mononegavirales: update 2017. Arch Virol 162:2493-2504

4. Aréchiga Ceballos N, Vázquez Morón S, Berciano JM, Nicolás O, Aznar López C, Juste J, Rodríguez Nevado C, Aguilar Setién A, Echevarría JE (2013) Novel lyssavirus in bat, Spain. Emerg Infect Dis 19:793-795

5. Bishop DHL, Pringle CR (1995) Order Mononegavirales. In: Murphy FA, Fauquet CM, Bishop DHL, Ghabrial SA, Jarvis AW, Martelli GP, Mayo MA, Summers MD (eds) Virus taxonomy-Sixth Report of the International Committee on Taxonomy of Viruses/ Archives of Virology Supplement 10. Springer, Vienna, pp 265-267

6. Easton AJ, Pringle CR (2011) Order Mononegavirales. In: King AMQ, Adams MJ, Carstens EB, Lefkowitz EJ (eds) Virus taxonomy-Ninth Report of the International Committee on Taxonomy of Viruses. Elsevier/Academic Press, London, pp 653-657

7. Fauver JR, Grubaugh ND, Krajacich BJ, Weger-Lucarelli J, Lakin SM, Fakoli LS III, Bolay FK, Diclaro JW II, Dabiré KR, Foy BD, Brackney DE, Ebel GD, Stenglein MD (2016) West African Anopheles gambiae mosquitoes harbor a taxonomically diverse virome including new insect-specific flaviviruses, mononegaviruses, and totiviruses. Virology 498:288-299

8. Goldberg TL, Bennett AJ, Kityo R, Kuhn JH, Chapman CA (2017) Kanyawara virus: a novel rhabdovirus infecting newly discovered nycteribiid bat flies infesting previously unknown pteropodid bats in Uganda. Sci Rep 7:5287

9. Gunawardena PS, Marston DA, Ellis RJ, Wise EL, Karawita AC, Breed AC, McElhinney LM, Johnson N, Banyard AC, Fooks AR (2016) Lyssavirus in Indian flying foxes, Sri Lanka. Emerg Infect Dis 22:1456-1459

10. Hyndman TH, Shilton CM, Stenglein MD, Wellehan JFX Jr (2018) Divergent bornaviruses from Australian carpet pythons with neurological disease date the origin of extant Bornaviridae prior to the end-Cretaceous extinction. PLOS Pathog 14:e1006881

11. Kuhn JH, Bekal S, Caì Y, Clawson AN, Domier LL, Herrel M, Jahrling PB, Kondo H, Lambert KN, Mihindukulasuriya KA, Nowotny N, Radoshitzky SR, Schneider U, Staeheli P, Suzuki N, Tesh RB, Wang D, Wang L-F, Dietzgen RG (2013) Nyamiviridae: proposal for a new family in the order Mononegavirales. Arch Virol 158:2209-2226

12. Lee H-J, Kim J-Y, Lee Y-J, Lee E-K, Song B-M, Lee H-S, Choi K-S (2017) A novel avian paramyxovirus (putative serotype 15) isolated from wild birds. Front Microbiol 8:786

13. Li C-X, Shi M, Tian J-H, Lin X-D, Kang Y-J, Chen L-J, Qin X-C, Xu J, Holmes EC, Zhang Y-Z (2015) Unprecedented genomic diversity of RNA viruses in arthropods reveals the ancestry of negative-sense RNA viruses. Elife 4:e05378

14. Longdon B, Murray GG, Palmer WJ, Day JP, Parker DJ, Welch JJ, Obbard DJ, Jiggins FM (2015) The evolution, diversity, and host associations of rhabdoviruses. Virus Evol 1:vev014

15. Marston DA, Ellis RJ, Wise EL, Aréchiga Ceballos N, Freuling CM, Banyard AC, McElhinney LM, de Lamballerie X, Müller T, Fooks AR, Echevarría JE (2017) Complete genome sequence of Lleida bat lyssavirus. Genome Announc 5:e01427

16. Marzano S-YL, Domier LL (2016) Novel mycoviruses discovered from metatranscriptomics survey of soybean phyllosphere phytobiomes. Virus Res 213:332-342

17. Marzano S-YL, Nelson BD, Ajayi-Oyetunde O, Bradley CA, Hughes TJ, Hartman GL, Eastburn DM, Domier LL (2016) Identification of diverse mycoviruses through metatranscriptomics characterization of the viromes of five major fungal plant pathogens. J Virol 90:6846-6863

18. Neira V, Tapia R, Verdugo C, Barriga G, Mor S, Ng TFF, García V, del Río J, Rodrigues P, Briceño C, Medina RA, 
González-Acuña D (2017) Novel avulaviruses in penguins, Antarctica. Emerg Infect Dis 23:1212-1214

19. Pringle CR (1991) Order Mononegavirales. In: Francki RIB, Fauquet CM, Knudson DL, Brown F (eds) Classification and nomenclature of viruses-Fifth Report of the International Committee on Taxonomy of Viruses/Archives of Virology Supplementum 2. Springer, Vienna, pp 239-241

20. Pringle CR, Alexander DJ, Billeter MA, Collins PL, Kingsbury DW, Lipkind MA, Nagai Y, Orvell C, Rima B, Rott R, ter Meulen V (1991) The order Mononegavirales. Arch Virol 117:137-140

21. Pringle CR (1996) Virus taxonomy 1996-a bulletin from the Xth International Congress of Virology in Jerusalem. Arch Virol 141:2251-2256

22. Pringle CR (1997) The order Mononegavirales — current status. Arch Virol 142:2321-2326

23. Pringle CR (2000) Order Mononegavirales. In: van Regenmortel MHV, Fauquet CM, Bishop DHL, Carstens EB, Estes MK, Lemon SM, Maniloff J, Mayo MA, McGeoch DJ, Pringle CR, Wickner RB (eds) Virus taxonomy-Seventh Report of the International Committee on Taxonomy of Viruses. Academic Press, San Diego, pp 525-530

24. Pringle CR (2005) Order Mononegavirales. In: Fauquet CM, Mayo MA, Maniloff J, Desselberger U, Ball LA (eds) Virus taxonomy-Eighth Report of the International Committee on
Taxonomy of Viruses. Elsevier/Academic Press, San Diego, pp 609-614

25. Shi M, Lin X-D, Tian J-H, Chen L-J, Chen X, Li C-X, Qin X-C, Li J, Cao J-P, Eden J-S, Buchmann J, Wang W, Xu J, Holmes EC, Zhang Y-Z (2016) Redefining the invertebrate RNA virosphere. Nature 540:539-543

26. Standfast HA, Dyce AL, St George TD, Muller MJ, Doherty RL, Carley JG, Filippich C (1984) Isolation of arboviruses from insects collected at Beatrice Hill, Northern Territory of Australia, 1974-1976. Aust J Biol Sci 37:351-366

27. Thampaisarn R, Bui VN, Trinh DQ, Nagai M, Mizutani T, Omatsu T, Katayama Y, Gronsang D, Le DHT, Ogawa H, Imai K (2017) Characterization of avian paramyxovirus serotype 14 , a novel serotype, isolated from a duck fecal sample in Japan. Virus Res 228:46-57

28. Thomazelli LM, de Araújo J, Fabrizio T, Walker D, Reischak D, Ometto T, Barbosa CM, Petry MV, Webby RJ, Durigon EL (2017) Novel avian paramyxovirus (APMV-15) isolated from a migratory bird in South America. PLoS One 12:e0177214

29. Wiley MR, Prieto K, Blasdell KR, Caì Y, Campos Lawson C, Walker PJ, Chiu CY, Palacios G, Kuhn JH (2017) Beatrice Hill virus represents a novel species in the genus Tibrovirus (Mononegavirales: Rhabdoviridae). Genome Announc 5:e01485-01416

\section{Affiliations}

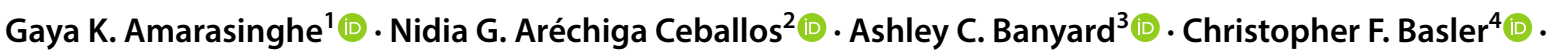
Sina Bavari ${ }^{5} \cdot$ Andrew J. Bennett $^{6} \cdot$ Kim R. Blasdell $^{7}$. Thomas Briese $^{8}\left(\right.$ Th $^{\circ} \cdot$ Alexander Bukreyev $^{9}$. Yíngyún Caì $^{10}$ (1) Charles H. Calisher ${ }^{11}$ [D . Cristine Campos Lawson ${ }^{10} \cdot$ Kartik Chandran $^{12}$ (D) . Colin A. Chapman ${ }^{13,14,15}$.

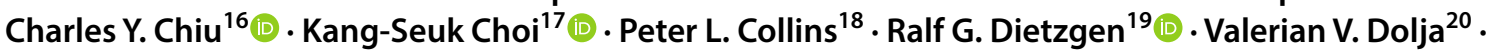

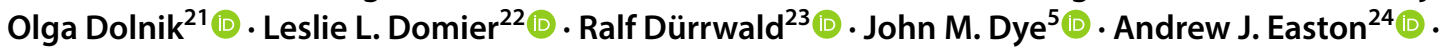

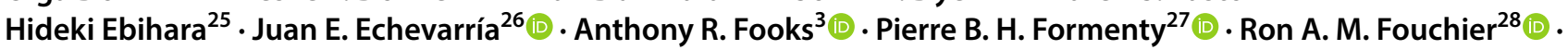
Conrad M. Freuling ${ }^{29}$ (D) Elodie Ghedin ${ }^{30}$ - Tony L. Goldberg ${ }^{6}$ (D) - Roger Hewson ${ }^{31}$ (i) $\cdot$ Masayuki Horie $^{32}$ (D) Timothy H. Hyndman ${ }^{33}$ (1) Dàohóng Jiāng ${ }^{34}$ - Robert Kityo ${ }^{35}$. Gary P. Kobinger ${ }^{36}$ - Hideki Kondō $^{37}$.

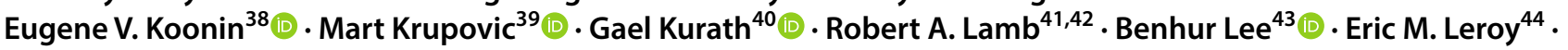

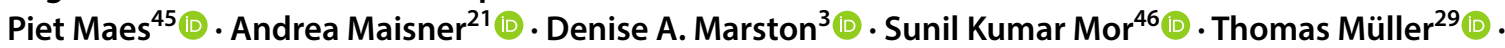

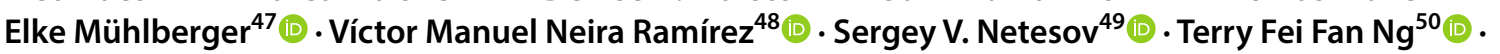

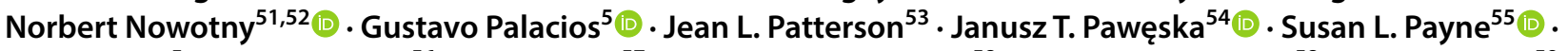

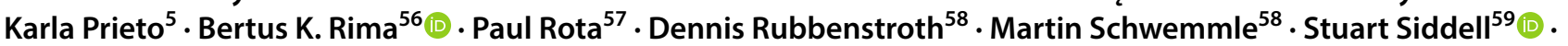
Sophie J. Smither ${ }^{60} \cdot$ Qisheng Song $^{61} \cdot$ Timothy Song $^{30} \cdot$ Mark D. Stenglein $^{62}$ (D) David M. Stone ${ }^{63}$ (D) Ayato Takada $^{64}\left(\right.$ Robert B. Tesh $^{65} \cdot$ Luciano Matsumiya Thomazelli $^{66}{ }^{6} \cdot$ Keizō Tomonaga $^{67} \cdot$ Noël Tordo $^{68,69}$.

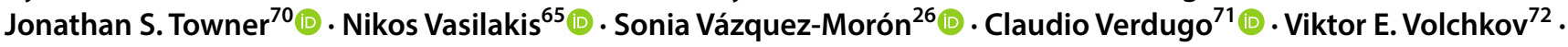
Victoria Wahl ${ }^{73}$ (1) Peter J. Walker ${ }^{74}$. David Wang ${ }^{75} \cdot$ Lin-Fa Wang $^{76} \cdot$ James F. X. Wellehan $^{77}$ (1)

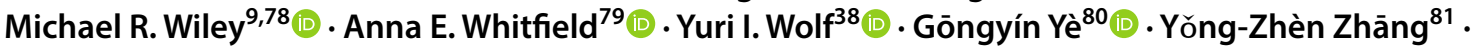
Jens H. Kuhn ${ }^{10}$ (1)

Jens H. Kuhn

kuhnjens@mail.nih.gov

1 Department of Pathology and Immunology, Washington University School of Medicine, St. Louis, MO, USA

2 Laboratorio de Rabia, Instituto de Diagnóstico y Referencias Epidemiológicos, Mexico City, Mexico

3 Animal and Plant Health Agency, Weybridge, Surrey, UK
4 Center for Microbial Pathogenesis, Institute for Biomedical Sciences, Georgia State University, Atlanta, GA, USA

5 United States Army Medical Research Institute of Infectious Diseases, Fort Detrick, Frederick, MD, USA

6 Department of Pathobiological Sciences, School of Veterinary Medicine, University of Wisconsin-Madison, Madison, WI, USA 
7 Australian Animal Health Laboratory, CSIRO Health and Biosecurity, Geelong, VIC, Australia

8 Department of Epidemiology, Center for Infection and Immunity, Mailman School of Public Health, Columbia University, New York, NY, USA

9 The University of Texas Medical Branch, Galveston, TX, USA

10 Integrated Research Facility at Fort Detrick (IRF-Frederick), Division of Clinical Research (DCR), National Institute of Allergy and Infectious Diseases (NIAID), National Institutes of Health (NIH), B-8200 Research Plaza, Fort Detrick, Frederick, MD 21702, USA

11 Arthropod-Borne and Infectious Diseases Laboratory, College of Veterinary Medicine and Biomedical Sciences, Colorado State University, Fort Collins, CO, USA

12 Department of Microbiology and Immunology, Albert Einstein College of Medicine, Bronx, NY, USA

13 Department of Anthropology and McGill School of Environment, McGill University, Montreal, QC, Canada

14 Wildlife Conservation Society, Bronx, NY, USA

15 Section of Social Systems Evolution, Primate Research Institute, Kyoto University, Kyoto, Japan

16 University of California, San Francisco, CA, USA

17 Avian Disease Research Division, Animal and Plant Quarantine Agency, Gimcheon, Republic of Korea

18 Respiratory Viruses Section, Laboratory of Infectious Diseases, National Institute of Allergy and Infectious Diseases, National Institutes of Health, Bethesda, MA, USA

19 Queensland Alliance for Agriculture and Food Innovation, The University of Queensland, St. Lucia, QLD, Australia

20 Department of Botany and Plant Pathology and Center for Genome Research and Biocomputing, Oregon State University, Corvallis, OR, USA

21 Institute of Virology, Philipps University Marburg, Marburg, Germany

22 Department of Crop Sciences, University of Illinois, Chicago, IL, USA

23 Robert Koch Institut, Berlin, Germany

24 School of Life Sciences, University of Warwick, Coventry, $\mathrm{UK}$

25 Department of Molecular Medicine, Mayo Clinic, Rochester, MN, USA

26 National Center of Microbiology, Carlos III Institute of Health, Majadahonda, Madrid, Spain

27 World Health Organization, Geneva, Switzerland

28 Department of Viroscience, Erasmus Medical Center, Rotterdam, The Netherlands

29 Institute of Molecular Virology and Cell Biology, Friedrich-Loeffler-Institute, WHO Collaborating Centre for Rabies Surveillance and Research, Greifswald-Insel Riems, Germany

30 Department of Biology, Center for Genomics and Systems Biology, New York University, New York, NY, USA
31 Public Health England, Porton Down, Salisbury, Wiltshire, UK

32 Hakubi Center for Advanced Research, Kyoto University, Kyoto, Japan

33 College of Veterinary Medicine, School of Veterinary and Life Sciences, Murdoch University, Murdoch, WA, Australia

34 State Key Laboratory of Agricultural Microbiology, The Provincial Key Lab of Plant Pathology of Húběi Province, College of Plant Science and Technology, Huázhōng Agricultural University, Wuhan, China

35 Department of Zoology, Makerere University, Kampala, Uganda

36 Department of Microbiology, Immunology and Infectious Diseases, Université Laval, Quebec City, Canada

37 Institute of Plant Science and Resources, Okayama University, Kurashiki, Japan

38 National Center for Biotechnology Information, National Library of Medicine, National Institutes of Health, Bethesda, MD, USA

39 Department of Microbiology, Institut Pasteur, Paris, France

40 US Geological Survey Western Fisheries Research Center, Seattle, WA, USA

41 Department of Molecular Biosciences, Northwestern University, Evanston, IL, USA

42 Howard Hughes Medical Institute, Northwestern University, Evanston, IL, USA

43 Department of Microbiology, Icahn School of Medicine at Mount Sinai, New York, NY, USA

44 Centre International de Recherches Médicales de Franceville, Institut de Recherche pour le Développement, Franceville, Gabon

45 Zoonotic Infectious Diseases Unit, KU Leuven, Leuven, Belgium

46 Department of Veterinary Population Medicine and Veterinary Diagnostic Laboratory, College of Veterinary Medicine, University of Minnesota, St. Paul, MN, USA

47 Department of Microbiology and National Emerging Infectious Diseases Laboratories, Boston University School of Medicine, Boston, MA, USA

48 Universidad de Chile Facultad de Ciencias Veterinarias y Pecuarias, Santiago, Chile

49 Novosibirsk State University, Novosibirsk, Novosibirsk Oblast, Russia

50 Department of Laboratory Medicine, University of California at San Francisco, San Francisco, CA, USA

51 Institute of Virology, University of Veterinary Medicine, Vienna, Austria

52 Department of Basic Medical Sciences, College of Medicine, Mohammed Bin Rashid University of Medicine and Health Sciences, Dubai, United Arab Emirates

53 Department of Virology and Immunology, Texas Biomedical Research Institute, San Antonio, TX, USA 
54 Center for Emerging Zoonotic and Parasitic Diseases, National Institute for Communicable Diseases of the National Health Laboratory Service, Sandringham, Johannesburg, Gauteng, South Africa

55 Department of Veterinary Pathobiology, College of Veterinary Medicine and Biomedical Sciences, Texas A\&M University, College Station, TX, USA

56 Centre for Experimental Medicine, School of Medicine, Dentistry and Biomedical Sciences, The Queen's University of Belfast, Belfast, Northern Ireland, UK

57 National Center for Immunization and Respiratory Diseases, Centers for Disease Control and Prevention, Atlanta, GA, USA

58 Institute of Virology, Medical Center - University of Freiburg, Faculty of Medicine, University of Freiburg, Freiburg, Germany

59 School of Cellular and Molecular Medicine, University of Bristol, Bristol, UK

60 CBR Division, Dstl, Porton Down, Salisbury, Wiltshire, UK

61 Division of Plant Sciences, College of Agriculture, Food and Natural Resources, University of Missouri, Columbia, MO, USA

62 Department of Microbiology, Immunology, and Pathology, College of Veterinary Medicine and Biomedical Sciences, Colorado State University, Fort Collins, CO, USA

63 Centre for Environment, Fisheries and Aquaculture Science, Weymouth, Dorset, UK

64 Division of Global Epidemiology, Hokkaido University Research Center for Zoonosis Control, Sapporo, Japan

65 Department of Pathology and Center for Biodefense and Emerging Infectious Diseases, Center for Tropical Diseases and Institute for Human Infections and Immunity, The University of Texas Medical Branch, Galveston, TX, USA

66 Institute of Biomedical Science, Universidade de São Paulo, São Paulo, Brazil

67 Institute for Frontier Life and Medical Sciences (inFront), Kyoto University, Kyoto, Japan
68 Institut Pasteur, Unité des Stratégies Antivirales, WHO Collaborative Centre for Viral Haemorrhagic Fevers and Arboviruses, OIE Reference Laboratory for RVFV and CCHFV, Paris, France Institut Pasteur de Guinée, Conakry, Guinea

70 Viral Special Pathogens Branch, Division of High-Consequence Pathogens Pathology, National Center for Emerging and Zoonotic Infectious Diseases, Centers for Disease Control and Prevention, Atlanta, GA, USA

71 Universidad Austral de Chile Facultad de Ciencias Veterinarias, Valdivia, Chile

72 Molecular Basis of Viral Pathogenicity, CIRI, INSERM U1111-CNRS, UMR5308, Université de Lyon, Université Claude Bernard Lyon 1, Ecole Normale Supérieure de Lyon, Lyon, France

73 National Biodefense Analysis and Countermeasures Center, Fort Detrick, Frederick, MD, USA

74 School of Biological Sciences, University of Queensland, St. Lucia, QLD, Australia

75 Departments of Molecular Microbiology and Pathology and Immunology, Washington University School of Medicine, St. Louis, MO, USA

76 Programme in Emerging Infectious Diseases, Duke-NUS Medical School, Singapore, Singapore

77 College of Veterinary Medicine, University of Florida, Gainesville, FL, USA

78 University of Nebraska Medical Center, Omaha, NE, USA

79 Department of Entomology and Plant Pathology, North Carolina State University, Raleigh, NC, USA

80 State Key Laboratory of Rice Biology and Ministry of Agriculture Key Laboratory of Molecular Biology of Crop Pathogens and Insects, Institute of Insect Sciences, Zhejiang University, Hangzhou, China

81 State Key Laboratory for Infectious Disease Prevention and Control, Collaborative Innovation Center for Diagnosis and Treatment of Infectious Diseases, Department of Zoonoses, National Institute for Communicable Disease Control and Prevention, Chinese Center for Disease Control and Prevention, Changping, Beijing, China 